\title{
Performances des vaches laitières autour de la rentrée à l'étable à l'automne : influence du type de ration de base et de la quantité de concentré offerte
}

\author{
J.-B. COULON, P. D'HOUR*, J.-P. GAREL *, M. PETIT ** \\ Avec la collaboration technique de Renée Lefalvre et J.N. Rampon * \\ INRA, Theix, Laboratoire de la Lactation, F 63122 Ceyrat \\ *INRA, Domaine Expérimental, F 15330 Marcenat \\ **INRA, Theix, Laboratoire de l'Elevage Bovin, F 63122 Ceyrat
}

\begin{abstract}
Résumé
Vingt-quatre vaches multipares en fin de lactation ont reçu, dès leur rentrée à l'étable, un régime composé de foin (lot $F$ ) complémenté normalement en aliment concentré (au-dessus de $8 \mathrm{~kg}$ de lait) ou d'ensilage d'herbe complémenté normalement (lot $\mathrm{EH}$ ) ou avec un apport supplémentaire d'aliment concentré au cours des 2 premières semaines (lot EHC). Tous les animaux recevaient par ailleurs $4 \mathrm{~kg}$ de regain. Les quantités de fourrages ingérées ont augmenté très rapidement après la rentrée à l'étable et ont atteint en $2^{\circ}$ semaine respectivement 15,4 et $13,0 \mathrm{~kg} \mathrm{MS} / \mathrm{j}$ chez les animaux recevant du foin (lot $\mathrm{F}$ ) ou de l'ensilage (lots $\mathrm{EH}$ et $\mathrm{EHC}$ ). La production laitière a diminué beaucoup plus fortement chez les animaux des lots $\mathrm{F}$ et $\mathrm{EH}(-2,2 \mathrm{~kg}$ de lait entre la dernière semaine de pâturage et la première semaine de stabulation) que chez ceux du lot EHC $(-0,6 \mathrm{~kg})(\mathrm{P}<0,01)$. Le taux butyreux a augmenté plus fortement dans le lot $\mathrm{EH}(+2,3 \mathrm{~g}$ p. 1000) que dans le lot $\mathrm{F}(+1,4 \mathrm{~g}$ p. 1000$)$ et le lot $\mathrm{EHC}(+0,6 \mathrm{~g}$ p. 1000$)$. Le taux protéique a beaucoup plus diminué chez les animaux recevant de l'ensilage $(-2,6 \mathrm{~g}$ p. 1000 en moyenne) que chez ceux recevant du foin $(-1,4 \mathrm{~g}$ p. 1000$)$. Le premier jour suivant la rentrée à l'étable, les animaux sont restés debout significativement plus longtemps que les jours suivants $(P<0,01)$. Les différentes origines possibles de ces modifications sont discutées.
\end{abstract}

Mots clés : vache laitière, rentrée à l'étable, composition du lait, quantités ingérées.

\section{Introduction}

La rentrée à l'étable à l'automne est une période délicate pour les vaches laitières en production. En plus du changement de régime alimentaire, les modifications de l'environnement y sont parfois très importantes, en particulier si les vaches sont hivernées en stabulation entravée. Lorsque la rentrée à l'étable est brutale, comme c'est souvent le cas en régions d'altitude, elle peut s'accompagner d'une diminution de la production laitière et d'une chute des taux de matières grasses et de protéines. 
Nous avons ainsi observé, 5 années successivement, chez des animaux en fin de lactation, que la rentrée à l'étable entraînait une chute anormale de la production journalière (de près de $2 \mathrm{~kg}$ ), qui n'a pas été compensée par la suite (Coulon et al., 1986). Ces problèmes peuvent être encore accrus chez les vaches ayant un haut niveau de production à la rentrée à l'étable, même lorsque celle-ci s'accompagne d'une transition alimentaire et environnementale (BURSTEDT, 1979). A notre connaissance, aucune étude spécifique n'a cependant été entreprise sur cette période.

L’objectif de cet essai a été d'une part de décrire l'évolution des quantités ingérées de fourrages conservés par les animaux dès la rentrée à l'étable, et d'autre part, parmi les différentes causes possibles de cette modification de l'évolution de la production laitière (nutritionnelles, comportementales, digestives, endocriniennes...), d'étudier certains aspects alimentaires. En particulier, nous avons voulu savoir s'il était possible d'éviter ou de limiter la diminution de production en améliorant de façon transitoire le niveau d'alimentation lors de la rentrée à l'étable. Pour cela, nous avons comparé 2 rations hivernales à base de foin ou d'ensilage d'herbe, et sur cette dernière 2 niveaux de complémentation énergétique.

\section{Matériel et méthodes}

\section{A. Animaux et alimentation}

Trois lots de 8 vaches multipares de races Montbéliarde (18) et Pie Noire (6) ayant vêlé au printemps $(236 \pm 44$ jours de lactation au 6-11, date de la rentrée à l'étable) ont été constitués sur la base de la race, de la production laitière, de l'état corporel, de l'état de gestation et du poids vif. Leur production laitière était comprise entre 12 et $20 \mathrm{~kg} / \mathrm{j}$ durant la semaine précédant la rentrée à l'étable.

Au pâturage les 24 vaches étaient conduites en rotation sur des parcelles voisines de l'étable. Elles recevaient de l'aliment concentré à raison de $1 \mathrm{~kg}$ par tranche de $2,5 \mathrm{~kg}$ de lait au-dessus de $12 \mathrm{~kg} / \mathrm{j}$.

Pendant les 15 jours qui ont précédé la rentrée à l'étable, les vaches ont reçu un foin de regain de prairie naturelle, distribué à volonté pendant la traite. Par la suite, ce même foin a été distribué vers $15 \mathrm{~h}$, à raison de $4 \mathrm{~kg} / \mathrm{j}$. Les animaux des 2 premiers lots (lots $\mathrm{EH}$ et $\mathrm{EHC}$ ) ont reçu en plus, dès la rentrée à l'étable, de l'ensilage d'herbe de prairie naturelle, distribué le matin (vers $7 \mathrm{~h} 30$ ), en quantités limitées les 2 premiers jours (respectivement 8 et $10 \mathrm{~kg}$ de $\mathrm{MS} / \mathrm{j}$ ) et à volonté par la suite. Parallèlement, les animaux du troisième lot (lot $F$ ) recevaient du foin de prairie naturelle déprimée, distribué le matin, en quantités limitées les 2 premiers jours (respectivement 10 et $11 \mathrm{~kg}$ de $\mathrm{MS} / \mathrm{j}$ ) et à volonté par la suite, pendant 3 semaines. Le foin a ensuite été remplacé (en 3 jours) par de l'ensilage d'herbe offert à volonté. Tous les animaux recevaient donc le même régime $(4 \mathrm{~kg}$ de regain + ensilage d'herbe à volonté) à partir de la $4^{\mathrm{e}}$ semaine de stabulation.

Pendant toute la durée de l'essai $(-2$ semaines avant la rentrée à +6 semaines après), les quantités de concentré distribuées, à même niveau de production, ont été identiques pour les animaux des lots $\mathrm{F}$ et $\mathrm{EH}$. La production laitière retenue pour le calcul de la complémentation a été celle des semaines -4 et -3 avant la rentrée 
à l'étable ; on a considéré que la ration de base satisfaisait les besoins énergétiques et azotés d'entretien plus ceux nécessaires à la production de $8 \mathrm{~kg}$ de lait et on a distribué l'aliment concentré à raison de $1 \mathrm{~kg}$ par tranche de $2,5 \mathrm{~kg}$ de lait au-dessus de cette production.

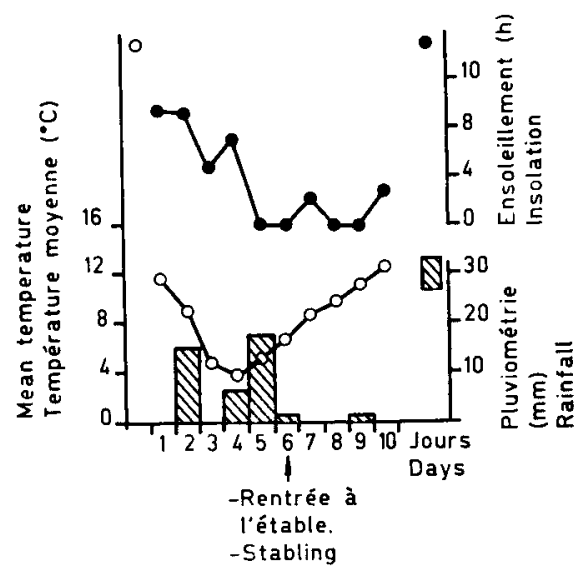

FIG. 1

Conditions météorologiques autour de la rentrée à l'étable.

Climate conditions around stabling.

TABleau 1

Composition et valeur nutritive des aliments.

Composition and nutritive value of feeds.

\begin{tabular}{|c|c|c|c|c|}
\hline & $\begin{array}{l}\text { Foin } \\
\text { Hay }\end{array}$ & $\begin{array}{c}\text { Regain } \\
\text { Aftermath }\end{array}$ & $\begin{array}{l}\text { Ensilage } \\
\text { d'herbe } \\
\text { Grass silage }\end{array}$ & $\begin{array}{c}\text { Concentré (1) } \\
\text { Concentrate }\end{array}$ \\
\hline $\begin{array}{l}\text { Date de récolte } \ldots \ldots \ldots \ldots \\
\text { Harvest time }\end{array}$ & $5 / 7$ & $31 / 8$ & $19 / 6$ & - \\
\hline $\begin{array}{l}\text { Teneur en matière sèche } \\
\text { (p. 100) . . . . . . . . . } \\
\text { Dry matter content }\end{array}$ & 85,4 & 85,2 & 23,6 & 88,0 \\
\hline $\begin{array}{l}\text { Matière organique (p. } 100 \mathrm{MS}) \\
\text { Organic matter }(\% \quad \text { DM) }\end{array}$ & 90,2 & 89,5 & 86,3 & 93,2 \\
\hline $\begin{array}{l}\text { Matières azotées totales } \\
\text { (p. } 100 \text { MS } \ldots \ldots \ldots \ldots \ldots \\
\text { Crude protein }(\% \text { DM })\end{array}$ & 16,2 & 13,8 & 15,9 & 18,5 \\
\hline $\begin{array}{l}\text { Cellulose brute (p. } 100 \mathrm{MS}) \\
\text { Crude fibre }(\% \text { DM) }\end{array}$ & 27,2 & 26,5 & 31,1 & 6,6 \\
\hline $\begin{array}{l}\text { Valeur nutritive }(\mathrm{kg} \mathrm{MS}) \\
\text { Nutritive value }(\mathrm{kg} \text { DM) } \\
\text { UFL } \ldots \ldots \ldots \ldots \ldots \ldots \ldots \ldots \\
\text { PDIN }(\mathrm{g}) \ldots \ldots \ldots \ldots \ldots \ldots \ldots \\
\text { PDIE }(\mathrm{g}) \ldots \ldots \ldots \ldots \ldots \ldots\end{array}$ & $\begin{array}{c}0,82 \\
100 \\
94\end{array}$ & $\begin{array}{l}0,67 \\
85 \\
82\end{array}$ & $\begin{array}{l}0,81 \\
95 \\
75\end{array}$ & $\begin{array}{l}1,05 \\
124 \\
123\end{array}$ \\
\hline
\end{tabular}

(1) Composition (p. 100) : céréales (40), pulpes de betteraves (30), tourteau tanné (22), mélasse (5), minéraux (3) - Composition (p. 100): cereals (40), beet pulp (30), treated oil meal (22), molasses (5), mineral mixture (3). 
Les animaux du lot $\mathrm{EHC}$ ont reçu, à partir de la veille de la rentrée à l'étable et durant 2 semaines, $2 \mathrm{~kg}$ de concentré de plus $(2,5 \mathrm{~kg}$ la première semaine et $1,5 \mathrm{~kg}$ la seconde) que les animaux des lots $\mathrm{F}$ et $\mathrm{EH}$. A partir de la $3^{\mathrm{c}}$ semaine, tous les animaux ont de nouveau reçu la même quantité d'aliment concentré. Un complément minéral $(10 \mathrm{P}-14 \mathrm{Ca})$ a été distribué à tous les animaux $(100 \mathrm{~g} / \mathrm{j})$ à partir de la rentrée à l'étable.

Jusqu'à ce jour (6-11), les animaux passaient la journée et la nuit (à l'exception des traites) au pâturage ; ils étaient amenés à l'étable (stabulation entravée, paillée) et attachés 2 fois par jour pour la traite qui durait environ $2 \mathrm{~h}$. Ils ne sont plus ressortis au-delà du soir du 6 novembre. Les conditions météorologiques des jours entourant la rentrée à l'étable sont précisées à la figure 1. La composition chimique et la valeur nutritive des aliments sont précisées au tableau 1. L'ensilage d'herbe utilisé avait été récolté directement à l'aide d'une machine double coupe réalisant des brins moyens à longs ( 5 à $10 \mathrm{~cm}$ ) et additionné de 3 litres d'acide formique par tonne de fourrage vert.

\section{B. Mesures}

Les quantités de lait produites ont été pesées tous les jours : le taux butyreux et le taux protéique ont été dosés à chaque traite, par spectrophotométrie infra-rouge, tous les jours de 5 jours avant à 5 jours après la rentrée à l'étable et 2 jours par semaine par la suite. Les quantités ingérées d'aliments conservés ont été mesurées tous les jours entre les jours -14 et +12 autour de la rentrée à l'étable et 5 jours par semaine ensuite. Les animaux ont été pesés 15 jours avant la rentrée à l'étable et toutes les semaines après; une double pesée et une notation d'état corporel (par maniement - note de 0 à 5 - BAzIN, 1984) ont été réalisées juste avant la rentrée à l'étable et 6 semaines après.

Quatre jours avant la rentrée à l'étable, 2 jours après et 3 semaines après, un échantillon de sang a été prélevé à la queue des animaux, le matin vers $8 \mathrm{~h}$, après la traite et avant la distribution des aliments. Sur le plasma obtenu par centrifugation et conservé au congélateur, on a dosé, à l'aide d'un appareil de dosage automatique ISAMAT, le glucose (méthode de Trinder, 1969), le 3-hydroxybutyrate (BHB) (méthode de Williamson \& Mellanby, 1974) et les acides gras non estérifiés (AGNE) (méthode de Trout et al., 1960).

Les 2 premiers jours, le $8^{\prime \prime}$ et le $15^{\prime \prime}$ jour après la rentrée à l'étable, on a observé (toutes les $5 \mathrm{mn}$ ), visuellement le jour et à l'aide d'une caméra la nuit, la position (debout ou couchée) des animaux, de manière à préciser l'influence de la rentrée à l'étable sur le «bien-être » des animaux, en considérant que leur position pouvait en être un indicateur.

Le traitement statistique des résultats a été réalisé par analyse de variance à un facteur (lot) ou à 2 facteurs (lot et période).

\section{Résultats}

\section{A. Production et composition du lait}

La production laitière a diminué entre la semaine précédant et celle suivant la rentrée à l'étable de façon beaucoup plus importante $(P<0,01)$ chez les animaux 
des lots $\mathrm{EH}$ et $\mathrm{F}$ (respectivement $-2,3$ et $-2,1 \mathrm{~kg}$ ) que chez ceux du lot $\mathrm{EHC}$ $(-0,6 \mathrm{~kg})$ (fig. 2 et tabl. 2). Cette diminution a été indépendante du niveau de production initial des animaux. Pendant la même période, le taux butyreux a augmenté de 2,3 et 1,4 g p. $1000 \mathrm{chez}$ les animaux des lots $\mathrm{EH}$ et $\mathrm{F}$ respectivement et de $0,6 \mathrm{~g}$ p. 1000 seulement chez ceux du lot EHC. Aucun de ces accroissements n'est cependant significatif de même que leur différence d'un lot à l'autre. Il existe une liaison positive et significative $(P<0,05)$ entre l'augmentation du taux butyreux et la diminution de production laitière. En définitive, la production de matières grasses du lait a beaucoup plus diminué chez les lots $\mathrm{EH}$ et $\mathrm{F}$ (respectivement -59 et $-70 \mathrm{~g} / \mathrm{j}$ entre la dernière semaine de pâturage et la première semaine de stabulation) que chez le lot $\operatorname{EHC}(-16 \mathrm{~g} / \mathrm{j})$. Le taux protéique a diminué fortement $(\mathrm{P}<0,01)$ à la rentrée à l'étable $(-2,2$ g p. 1000$)$ mais beaucoup plus $(P<0,05)$ chez les animaux recevant de l'ensilage (respectivement $-2,8$ et $-2,4 \mathrm{~g} \mathrm{p} .1000$ pour les lots EH et EHC) que chez ceux recevant du foin $(-1,4$ g p. 1000$)$. La production de protéines a ainsi diminué de 95, 117 et $60 \mathrm{~g} / \mathrm{j}$ chez les vaches des lots F, EH et EHC respectivement. Malgré des différences importantes des taux butyreux et protéiques d'un animal à l'autre et d'un lot à l'autre au cours de la dernière semaine de pâturage, les variations de ce taux à la rentrée à l'étable ont été indépendantes de leur niveau avant la rentrée.

Que ce soit pour la production laitière ou pour les taux butyreux et protéique, l'essentiel des variations observées a eu lieu dans les 5 jours suivant la rentrée à l'étable (fig. 2). La production laitière s'est ensuite pratiquement stabilisée chez les vaches des lots $F$ et $E H$. Chez ces dernières, les fluctuations de production ont cependant été un peu plus importantes. Le changement de ration de base pour les animaux du lot $\mathrm{F}$ en $3^{\circ}$ semaine de stabulation n'a été accompagné d'aucune modification de la production laitière ; les taux protéique et surtout butyreux ont par contre augmenté sensiblement mais de manière passagère, de sorte que, à la fin de l'essai les écarts de taux observés d'un lot à l'autre étaient semblables à ceux observés pendant la dernière semaine de pâturage. La suppression de l'apport supplémentaire de concentré en fin de $2^{\mathrm{e}}$ semaine de stabulation chez les animaux du lot EHC n'a pas modifié l'évolution de leur production laitière qui est donc restée un peu supérieure (d'environ $1 \mathrm{~kg} / \mathrm{j}$ ) à celle des autres lots. En définitive chez ces animaux, l'apport supplémentaire de $28 \mathrm{~kg}$ de concentré juste après la rentrée s'est ainsi traduit par une production supplémentaire sur les 6 premières semaines de stabulation, de respectivement 59 et $51 \mathrm{~kg}$ par rapport aux lots $\mathrm{EH}$ et $\mathrm{F}$, soit une efficacité globale de l'aliment concentré voisine de $2 \mathrm{~kg}$ de lait par $\mathrm{kg}$ de concentré supplémentaire.

\section{B. Quantités d'aliments ingérées}

Au cours des 15 derniers jours de pâturage, les animaux ont ingéré en moyenne $3,1 \mathrm{~kg} \mathrm{MS} / \mathrm{j}$ de foin et $2,5 \mathrm{~kg} \mathrm{MS} / \mathrm{j}$ d'aliment concentré. A partir de la rentrée à l'étable ils ont accru très rapidement leur ingestion de fourrages conservés qui a été maximale dès la fin de la $1^{\text {re }}$ semaine pour les animaux recevant de l'ensilage $(13,0 \mathrm{~kg} \mathrm{MS} / \mathrm{j})$ et en milieu de $2^{\circ}$ semaine pour ceux recevant du foin $(15,4 \mathrm{~kg} \mathrm{MS} / \mathrm{j})$. Au cours de ces 2 premières semaines, les animaux du lot $\mathrm{EHC}$ bien que recevant $2 \mathrm{~kg}$ de concentré (1,74 $\mathrm{kg}$ de MS) de plus, ont consommé autant de fourrage que ceux du lot EH. Au cours des $2^{e}$ et $3^{e}$ semaines de stabulation, et alors que les consommations de fourrages étaient stabilisées, les animaux recevant uniquement du foin (lot $F$ ) ont ingéré au total $1,8 \mathrm{~kg}$ de $\mathrm{MS} / \mathrm{j}$ de plus que ceux recevant de l'ensilage à même niveau de 


\section{TABLEAU 2}

Variations des différents paramètres zootechniques et métaboliques à la rentrée à l'étable.

Variations in the animal performance and in metabolite concentrations at stabling.

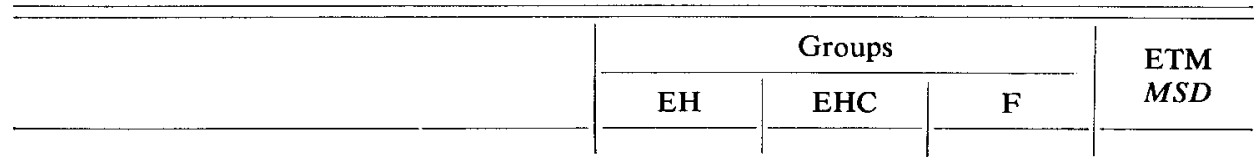

2 dernières semaines de pâturage

Last 2 weeks on pasture

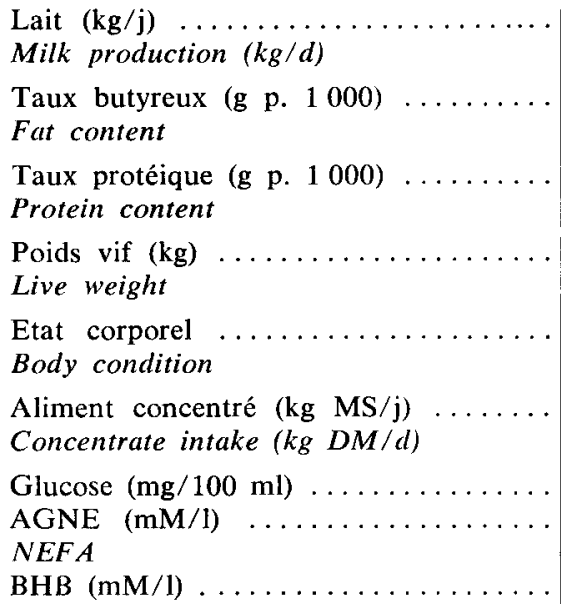

\begin{tabular}{c|c|c|c}
15,7 & 16,0 & 15,6 & 2,6 \\
39,2 & 37,9 & 41,7 & 4,6 \\
35,1 & 35,7 & 36,9 & 3,3 \\
629 & 622 & 607 & 49 \\
2,04 & 2,26 & 1,69 & 0,5 \\
2,55 & 2,56 & 2,58 & 1,00 \\
69,6 & 72,3 & 67,4 & 3,1 \\
0,15 & 0,14 & 0,15 & 0,02 \\
0,65 & 0,52 & 0,54 & 0,15
\end{tabular}

Différence entre la première semaine de stabulation et la dernière semaine de pâturage Difference between the first week indoors and the last week on pasture

\begin{tabular}{|c|c|c|c|c|}
\hline $\begin{array}{l}\text { Lait } \ldots \ldots \ldots \ldots \ldots \ldots \ldots \ldots \ldots \\
\text { Milk production }\end{array}$ & $-2,3 a$ & $-0,6 b$ & $-2,1 \mathrm{a}$ & 1,2 \\
\hline $\begin{array}{l}\text { Taux butyreux } \ldots \cdots \cdots \cdots \cdots \cdots \cdots \\
\text { Fat content }\end{array}$ & $+2,3$ & $+0,6$ & $+1,4$ & 2,6 \\
\hline $\begin{array}{l}\text { Taux protéique } \ldots \ldots \ldots \ldots \ldots \ldots \ldots \ldots \\
\text { Protein content }\end{array}$ & $-2,8 \mathrm{a}$ & $-2,4 a$ & $-1,4 b$ & 1,1 \\
\hline $\begin{array}{l}\text { Poids } \text { vif } \ldots \ldots \ldots \ldots \ldots \ldots \ldots \ldots \ldots \\
\text { Live weight }\end{array}$ & +6 & +7 & -3 & 19 \\
\hline $\begin{array}{l}\text { Glucose } \ldots \ldots \ldots \ldots \ldots \ldots \ldots \ldots \ldots \ldots \ldots \\
\text { AGNE } \\
N E F A\end{array}$ & $\begin{array}{l}-2,6 \\
+0,28\end{array}$ & $\begin{array}{l}-4,6 \\
+0,23\end{array}$ & $\begin{array}{l}-1,5 \\
+0,29\end{array}$ & $\begin{array}{l}4,7 \\
0,11\end{array}$ \\
\hline $\mathrm{BHB} \quad \ldots \ldots \ldots \ldots \ldots \ldots \ldots \ldots \ldots \ldots \ldots \ldots$ & $-0,20$ & $-0,13$ & $-0,10$ & 0,15 \\
\hline
\end{tabular}

(1) Ecart-type moyen - Mean standard deviation.

Les valeurs suivies de lettres différentes sont significativement différentes $(\mathrm{P}<0,01)-$ Values with different letters are significantly different $(P<0.01)$. 


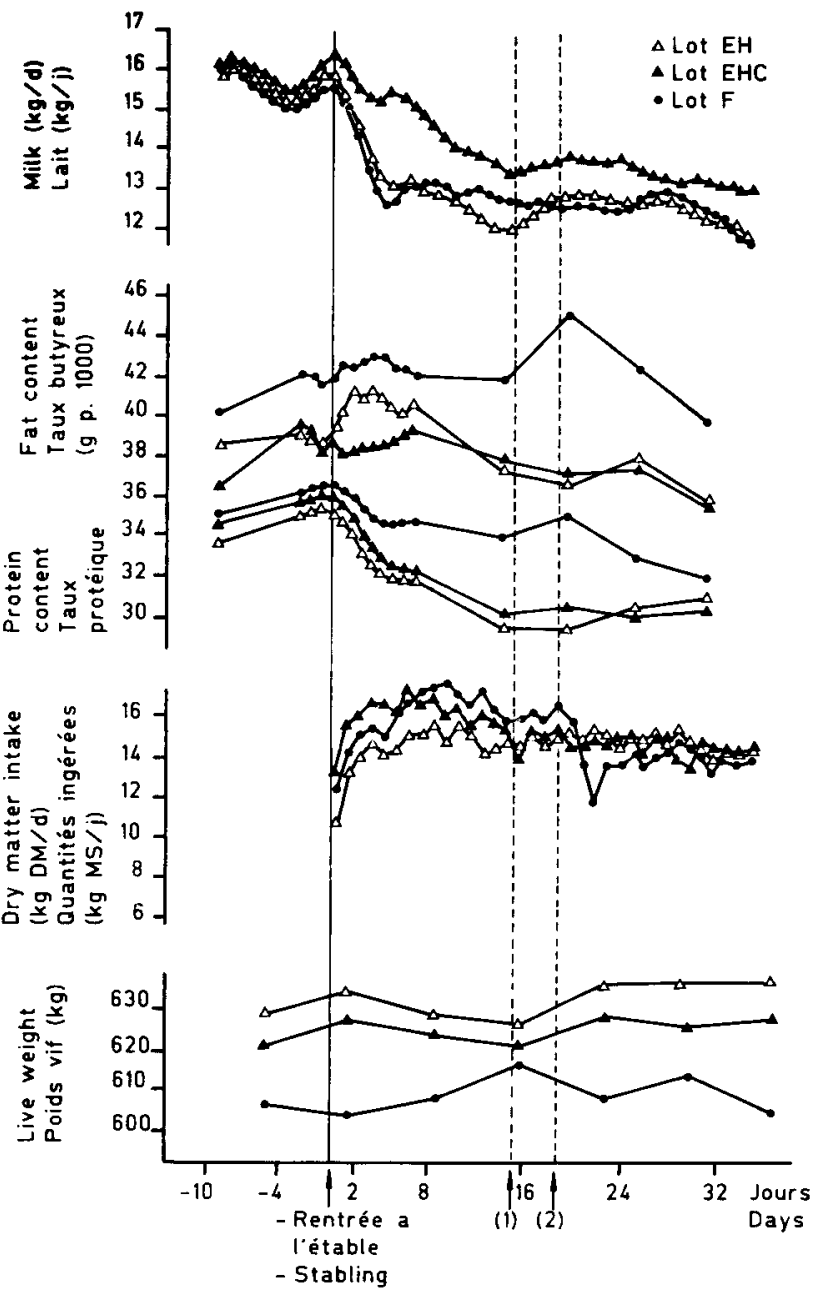

FIG. 2

Evolution de la production et de la composition du lait, des quantités ingérées et $d u$ poids vif. (La production laitière et les taux butyreux et protéique entre les jours -4 et +8 , sont des moyennes mobiles sur 5 jours).

Variation in milk production, milk composition, dry matter intake and live-weight. (Milk production, fat content and protein content between days -4 and +8 , are moving averages on 5 days)

(1) Arrêt de l'apport supplémentaire de concentré au lot EHC.

End of supplementary concentrate supply to group EHC.

(2) Changement de ration de base pour le lot F.

Change of roughage in group $F$. 
complémentation (lot $\mathrm{EH})(\mathrm{P}<0,01)$; les quantités totales d'aliments ingérées étaient alors respectivement de 2,77 et $2,39 \mathrm{~kg}$ de $\mathrm{MS} / 100 \mathrm{~kg}$ de poids vif pour une ration composée de 15 à 18 p. 100 d'aliment concentré. En fin de $3^{\text {" semaine de }}$ stabulation le changement de ration de base pour les animaux du lot $\mathrm{F}$ s'est accompagné d'une chute importante mais passagère des quantités ingérées (fig. 2) ; celles-ci sont en effet rapidement remontées à un niveau identique à celui des 2 autres lots $(12,0 \mathrm{~kg} / \mathrm{j}$ de $M S$ de fourrage $)$.

\section{Poids vif et état corporel}

Entre la dernière semaine de pâturage et la $6^{\circ}$ semaine de stabulation les animaux des lots $\mathrm{EH}$ et $\mathrm{EHC}$ ont gagné $7 \mathrm{~kg}$ de poids vif et 0,4 point d'état, alors que ceux $\mathrm{du}$ lot $\mathrm{F}$ perdaient $3 \mathrm{~kg}$ de poids vif mais gagnaient 0,7 point d'état. Ces différences (d'un lot à l'autre et d'une période à l'autre) ne sont cependant pas significatives. Ce sont les animaux dont la baisse de production au cours de l'essai a été la moins importante qui ont repris le plus d'état corporel $(R=0,46 ; P<0,05)$, mais ce sont aussi ceux qui ont présenté les niveaux d'ingestion les plus élevés $(R=0,38$; $\mathrm{P}>0,05)$.

\section{Métabolites sanguins}

Au pâturage, les teneurs plasmatiques des métabolites que nous avons dosés étaient voisines d'un lot à l'autre et égales respectivement, en moyenne, à $69,8 \mathrm{mg} /$ $100 \mathrm{ml}, 0,57 \mathrm{mM} / 1$ et $0,15 \mathrm{mM} / 1$ pour le glucose, le BHB et les AGNE. La rentrée à l'étable s'est accompagnée d'une légère mais significative $(P<0,01)$ diminution des teneurs en BHB $(-0,15 \mathrm{mM} / \mathrm{l})$, un peu plus importante $(\mathrm{P}>0,05)$ chez les animaux du lot $\mathrm{EH}(-0,20 \mathrm{mM} / \mathrm{l})$ que chez ceux des lots $\mathrm{EHC}$ et $\mathrm{F}$ (respectivement $-0,13$ et $-0,10 \mathrm{mM} / 1)$ et d'une diminution des teneurs en glucose $(-3 \mathrm{mg} / 100 \mathrm{ml})$ $(P<0,01)$ là aussi légèrement plus importante $(P>0,05)$ chez les animaux recevant de l'ensilage (respectivement $-2,6 \mathrm{mg} / \mathrm{ml},-4,6$ et $-1,5$ pour les lots $\mathrm{EH}$, EHC et F). Mais ce sont surtout les AGNE qui ont présenté les variations les plus importantes $(+0,27 \mathrm{mM} / 1, \mathrm{P}<0,01)$ quel que soit le lot. Trois semaines après la rentrée ils étaient revenus à un niveau faible $(0,10 \mathrm{mM} / \mathrm{l})$, tandis que les teneurs en glucose augmentaient (respectivement $+9,4,+9,4$ et $+4,1 \mathrm{mg} / 100 \mathrm{ml}$ chez les lots $\mathrm{EH}, \mathrm{EHC}$ et $F$ ) et que celles en BHB évoluaient peu.

\section{E. Comportement à l'étable}

Les 24 premières heures qui ont suivi la rentrée à l'étable, les animaux sont restés debout 70 p. 100 du temps, contre seulement 50 p. 100 le 2" jour, le 8" jour ou le $15^{\circ}$ jour $(P<0,01)$. Cette différence se crée essentiellement la nuit entre $18 \mathrm{~h}$ et $7 \mathrm{~h})\left(64 \mathrm{p} .100 \mathrm{du}\right.$ temps passé debout la $1^{\text {re }}$ nuit contre 35 p. 100 les nuits suivantes), mais aussi le jour $(7 \mathrm{~h}-18 \mathrm{~h}$ ) (76 p. $100 \mathrm{du}$ temps passé debout contre 66 p. 100 ; $P<0,01)$. Quelle que soit la nuit d'observation il n'y a pas eu de différence significative d'un lot à l'autre. Nous n'avons pas observé de liaison interindividuelle entre le temps passé debout la première nuit et la chute de production laitière à la traite du matin (par rapport à celle du matin précédent). 


\section{Discussion - Conclusion}

Dans les quelques jours qui ont suivi la rentrée à l'étable les vaches ont été capables d'ingérer des quantités élevées de matière sèche, en particulier celles du lot $\mathrm{F}$ $(2,8 \mathrm{~kg} \mathrm{MS} / 100 \mathrm{~kg}$ de poids vif), comparables aux valeurs observées à stade de lactation et niveaux de production équivalents chez des animaux recevant depuis plusieurs semaines des rations principalement composées de foin (Trimberger et al., 1963) ou d'ensilage d'herbe (ETTAla \& Lampila, 1978). Ces niveaux élevés d'ingestion ont été permis par l'excellente qualité des fourrages offerts, en particulier du foin. Ils ont peut-être été favorisés par : 1) leur distribution en petite quantité avant la rentrée à l'étable ; 2) l'âge et le format élevés des animaux, ainsi que leur état d'engraissement médiocre, dont on sait, tout du moins en début de lactation (Garnsworthy \& Topps, 1982) ou en fin de gestation (Bines \& Morant, 1983), qu'il stimule l'ingestion; 3) l'habitude qu'avaient les animaux de rentrer 2 fois par jour à l'étable pour la traite qui a pu limiter le "stress" lié au changement d'environnement. D'ailleurs, il semble que les vaches n'aient été perturbées (si l'on en juge par le temps passé debout) que le $1^{\text {ar }}$ jour suivant la rentrée.

Mais en dépit de l'augmentation rapide des quantités de fourrages ingérées, la diminution de la production laitière (voisine de celle que nous avions observée précédemment - Coulon et al., 1986), celle des taux de glucose plasmatique et l'augmentation des taux d'AGNE suggèrent que les apports énergétiques disponibles au niveau de la mamelle ont été fortement réduits à la rentrée à l'étable. Or, ces apports, calculés en adoptant pour les fourrages les valeurs obtenues à partir des mesures de leur digestibilité sur moutons (tabl. 1), et en prenant pour l'entretien les besoins proposés par VÉRITÉ et al. (1978), auraient dû permettre des productions de 16, 20 et $19 \mathrm{~kg}$ de lait 4 p. 100 au cours des 2 premières semaines pour les lots EH, EHC et F respectivement. Ce calcul a donc surestimé la valeur énergétique des rations de près de 20 p. 100 . Plusieurs raisons peuvent être avancées pour expliquer cette discordance : 1) La digestibilité des fourrages a été surévaluée par la mesure sur moutons, bien que ce soit souvent l'inverse que l'on observe (DuLPHY \& CARLE, données non publiées). 2) Le changement de nature des aliments à la rentrée à l'étable a entraîné une baisse momentanée de la digestibilité en raison de la nécessaire adaptation des microorganismes du rumen à un nouveau régime, qui durerait 10 à 15 jours (MOSELEY et al., 1976 ; Kaufmann et al., 1980). Dans cette hypothèse, il est cependant étonnant que les quantités ingérées aient été si rapidement importantes; on sait en effet qu'une baisse de l'efficacité de la digestion dans le rumen limite généralement l'ingestion (JARRIGE et al., 1978). 3) Enfin, il est aussi possible que nous ayons sous-estimé les besoins énergétiques d'entretien des animaux, au moins les premiers jours qui ont suivi la rentrée. On sait en effet que ces besoins sont supérieurs d'au moins 20 p. 100 au pâturage (JARRIGE et al., 1978), et il est possible qu'ils ne reviennent pas à leur niveau « hivernal » immédiatement après la rentrée (VERMOREL, communication personnelle).

Ces raisons, même si elles peuvent être en partie exactes, ne sont peut-être cependant pas suffisantes pour qu'on puisse en conclure que les vaches étaient en bilan énergétique aussi négatif que ne le suggère la diminution de production laitière. On peut en effet aussi penser que la rentrée à l'étable s'est accompagnée d'une modification de l'utilisation des nutriments, soit que la mamelle ait été moins apte à les capter, soit que le tissu adipeux ait été davantage prioritaire que pendant la période 
de pâturage. Cette modification serait à l'origine du gain d'état corporel que nous avons observé. Elle a pu avoir lieu dès la rentrée, et être alors la cause de la diminution de production laitière, ou quelques jours plus tard et en être la conséquence. Dans la première hypothèse, cette modification de la répartition des nutriments pourrait être due au changement de nature du régime, bien que le foin et l'ensilage d'herbe soient des rations connues pour leur bonne valeur laitière (VÉRITÉ, 1983), mais peut-être aussi à des modifications hormonales entraînées par le changement d'environnement (DANTzer \& MoRMEDE, 1979), en particulier de la cortisolémie (McNATTY \& Thurley, 1973) dont l'augmentation a parfois été mise en relation avec des baisses de production laitière (JoHNSON \& VANJONACK, 1976). Dans la seconde hypothèse, une fois la période d'adaptation réalisée, les animaux n'ont pas pu augmenter leur production laitière, vraisemblablement en raison de leur stade avancé de lactation et de gestation, l'énergie disponible étant alors utilisée pour la reconstitution des réserves corporelles.

En définitive, cet essai a permis de montrer que la chute de production laitière à la rentrée à l'étable était importante lorsque l'on complémentait les vaches de manière normale ; qu'elle était du même ordre de grandeur, que l'on utilise une ration mixte (foin plus ensilage d'herbe) ou à base de foin seul ; que l'on pouvait vraisemblablement la limiter en les complétant plus libéralement : la production laitière du lot EHC en $6^{\circ}$ semaine de stabulation n'a en effet été inférieure que de $0,5 \mathrm{~kg}$ à celle prévisible à partir du niveau de la semaine précédant la rentrée (en admettant une décroissance hebdomadaire de 2,5 p. 100) ; qu'elle n'était pas due à une ingestion trop faible mais plutôt à une mauvaise utilisation des aliments ingérés pour la production laitière. Des études sont donc nécessaires pour préciser les raisons de cette mauvaise utilisation.

Reçu en octobre 1985.

Accepté en janvier 1986.

\begin{abstract}
Summary
Dairy cow performance at stabling in autumn : influence of the nature of roughage and of the level of concentrate supplementation
\end{abstract}

Between the last two weeks of pasture and the first six weeks after stabling, 24 multiparous cows in late lactation were given a diet composed of hay (group F) normally supplemented with concentrate (above $8 \mathrm{~kg}$ milk yield) or grass silage either normally supplemented (group EH) or with an additional amount of concentrate $(2 \mathrm{~kg} / \mathrm{d}$ ) (group EHC). Moreover, all the animals were given aftermath $(4 \mathrm{~kg} / \mathrm{d})$. Roughage dry matter intake increased very rapidly after stabling and reached during the second week 15.4 and $13.0 \mathrm{~kg} / \mathrm{d}$, respectively in cows receiving hay (group F) and in those receiving silage (groups EH and EHC) (fig. 1). Milk yield decreased much more markedly in groups $F$ and $\mathrm{EH}(-2.2 \mathrm{~kg}$ milk between the last week outdoors and the first week indoors) than in group EHC ( $-0.6 \mathrm{~kg})$ $(\mathrm{P}<0.01)$. Milk fat content increased more in group EH $(+2.3$ g p. 1000$)$ than in group $F$ $(+1.4$ g p. 1000$)$ and EHC $(+0.6$ g p. 1000$)$. Protein content decreased much more in animals fed silage $(-2.6 \mathrm{~g}$ p. 1000 on an average) than in those fed hay $(-1.4 \mathrm{~g} \mathrm{p} .1000)$. The first day following stabling, cows remained standing longer than the following days $(17 \mathrm{~h}$ vs 12$)(\mathrm{P}<0.01)$. The possible causes of these changes are discussed.

Key words : Dairy cow, stabling, milk composition, intake. 


\section{Références bibliographiques}

BAZIN S., 1984. Grille de notation de l'état d'engraissement des vaches Pies Noires. Ed. I.T.E.B., 149, rue de Bercy, 75595 Paris Cedex 12.

Bines J.A., Morant S.V., 1983. The effects of body condition on metabolic changes associated with intake of food by the cow, Br.J. Nutr., 50, 81-89.

BURSTEDT E., 1979. Some aspects of the nutritive value of grazed grass and carry over effects in the autumn. In : "European grazing workshop》, Lelystad, avril 1979.

Coulon J.B., Garel J.P., Hoden A., 1986. Evolution de la production et de la composition du lait à la rentrée à l'étable en fonction du type de ration hivernale. Bull. Tech. C.R.Z.V. Theix, INRA, 63, à paraître.

Dantzer R., Mormede P., 1979. Le stress en élevage intensif. Ed. Masson, France. 118 p.

Ettala E., Lampila M., 1978. Factors affecting voluntary silage intake by dairy cows. Ann. Agric. Fenn., 17, 163-174.

Garnsworthy P.C., ToOps J.H., 1982. The effect of body condition of dairy cows at calving on their food intake and performance when given complete diets. Anim. Prod., 35, 113-119.

Jarrige R., Gueguen L., Vermorel M., 1978. Entretien. In : «Alimentation des ruminants». 207-216. Ed. INRA Publications, route de Saint-Cyr, 78000 Versailles.

Johnson H.D., Vanjonack W.J., 1976. Effects of environmental and other stressors on blood hormone patterns in lactating animals. J. Dairy Sci., 59, 1603-1617.

Kaufmann W., Hagemeister H., Dirksen G., 1980. Adaptation to changes in dietary Composition, level and frequency of feeeding. In : Y. Ruckebusch \& P. Thivend (Eds.), Digestive physiology and metabolism in ruminants. Proc. 5th intern. symposium, Clermont-Ferrand, France, p. 587-602 (Lancaster, England : M.T.P. Press).

MaC Natty K.P., Thurley D.C., 1973. The episodic nature of changes in ovine plasma cortisol levels and their response to adrenaline during adaptation to a new environment. J. Endocrinol., 59, 171-181.

Moseley J.E., Coppock C.E., LAKE G.B., 1976. Abrupt changes in forage-concentrate ratios of complete feeds fed ad libitum to dairy cows. J. Dairy Sci., 59, 1471-1983.

Trimberger G.W., Gray H.G., Johnson W.L., Wright M.J., Vanvleck L.D., Henderson C.R., 1963. Forage appetite in dairy cattle. Proc. Cornell Conf. Feed. Manuf., 33-43.

Trinder P., 1969. Determination of glucose in blood using glucose oxydase with an alternative glucose acceptor. Ann. Clin. Biochem., 6, 24-27.

Trout D.L., Estes E.H., Friedberg S.J., 1960. Titration of free fatty acids of plasma : a study of current methods and new modification. J. Lipid. Res., 1, 199-202.

VÉRITÉ R., 1983. Valeur laitière des rations : Observations sur vaches. In «Quels aliments concentrés pour les vaches fortes productrices de lait», C.A.A.A., I.N.A., ParisGrignon, 13 octobre 1983.

Vérité R., Journet M., Gueguen L., Hoden A., 1978. Vaches laitières. In : «Alimentation des ruminants », 345-376. Ed. INRA Publications, route de Saint-Cyr, 78000 Versailles.

Williamson D.H., Mellanby Y., 1974. D (-) 3-hydroxybutyrate. In : H.V. Bergmeyer (ed.), Methods of enzymatic analysis, Vol. 4, 1836-1839 (Academic Press. New York, $2^{*}$ édition). 